Historic, Archive Document

Do not assume content reflects current scientific knowledge, policies, or practices. 


\section{Wholesale Trade-List - Season 1920-21}

\section{Gardens of the Blue Ridge E. C. ROBBINS}

\section{Collector and Plantsman}

Nearly Thirty Years' Experience with one of the Big Nurseries, Growing and Handling Native Ornamentals Exclusively

\section{Pineola, Avery Co., N. C.}

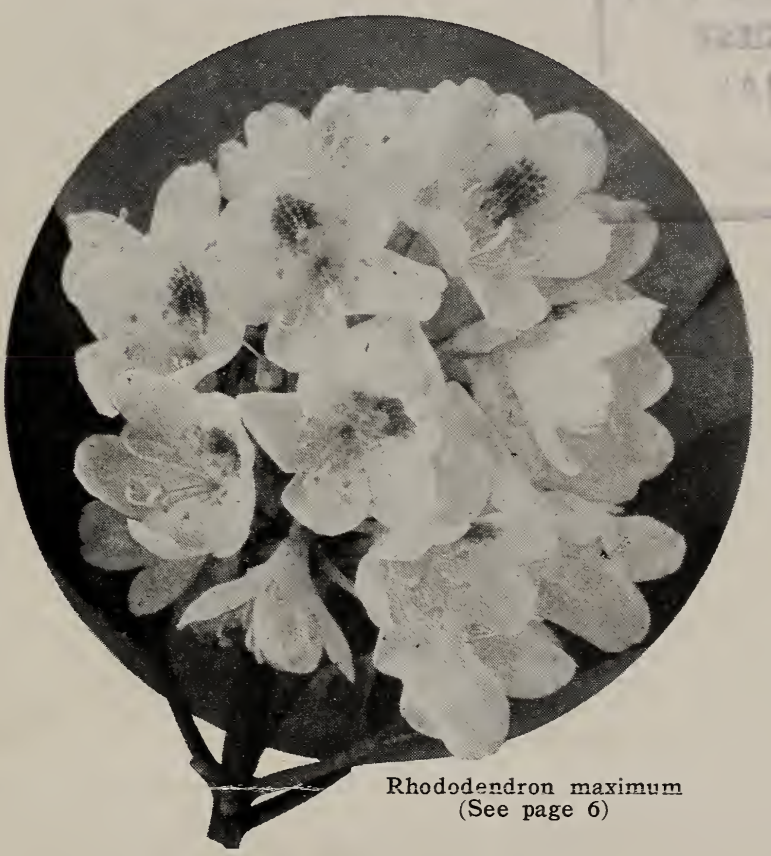

A Select Wholesale Price-List of Hardy and Rare Broad-Leaved Evergreens, Ornamental Trees, Shrubs, Vines, Ferns, and Herbaceous Plants of the Blue Ridge Mountains

MANY ITEMS NOT HEREIN LISTED CAN BE FURNISHED. CAN SUPPLY NEARLY ANY PLANT IN ITS PROPFR SEASON. COLLECTIONS TO ORDFR IN CARLOAD LOTS A SPECIALTY AT SPECIAL PRICES 


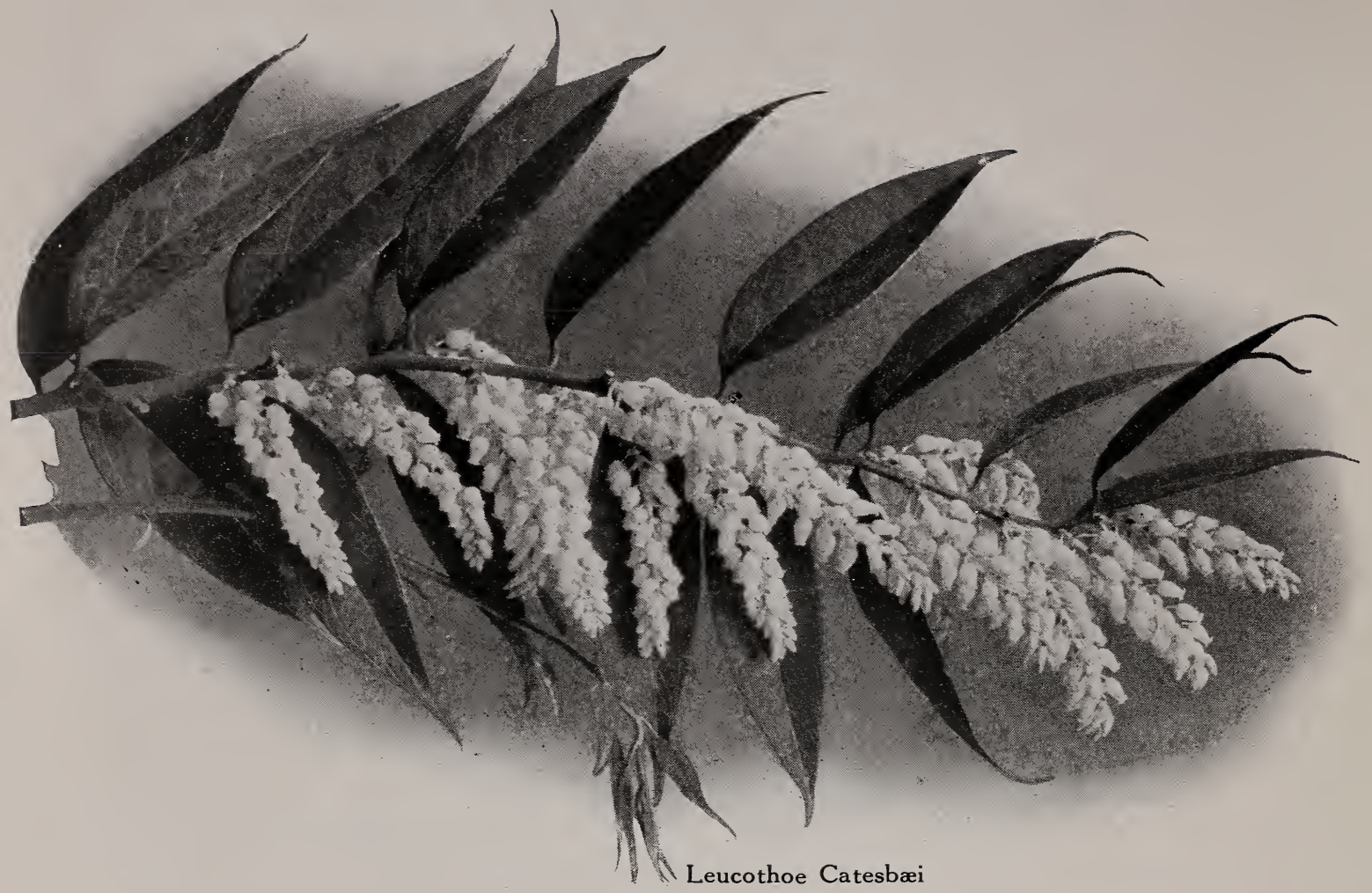

\section{NOTE TO CUSTOMERS}

\section{MY LOCATION}

Is on the summit of the Blue Ridge Mountains, "the most beautiful mountain region of the continent," in western North Carolina, nearly 4,000 feet above sea-level. I have no connection with any other nursery, and my business headquarters is Pineola, Avery County, North Carolina, U. S. A.

All offers of stock are made subject to conditions beyond my control

\section{PACKING AND GRADING}

Will be done in the most careful way. Boxing and burlapping charged at cost. Certificate of Inspection attached to all shipments.

\section{QUOTATIONS}

This list cancels previous offers and prices quoted are net and not subject to discount except by special agreement.

\section{REMITTANCE OR REFERENCE}

Should accompany orders from unknown correspondents. Discount of 3 per cent on cash orders. All bills due and payable 30 days from date of invoice, unless otherwise arranged.

\section{SHIPPING INSTRUCTIONS}

Should always be given fully, unless you prefer to leave to our best judgment. In this we assume no risk, our responsibility ceasing upon receipt of signed bill of lading by forwarders. All claims for damages in transit must be handled by consignee.

\section{CLAIMS}

All claims for errors must be reported upon receipt of goods and will be rectified promptly. Unless this is done I can accept no responsibility of any kind.

\section{ALL ORDERS}

Are accepted and filled on these terms. 


\section{DECIDUOUS TREES AND SHRUBS}

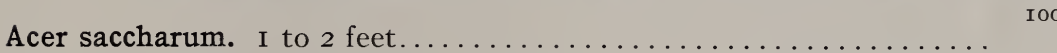

1,000

$\$ 2500$

5000

7500

3 to 4 feet.

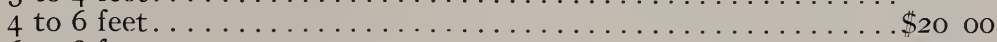

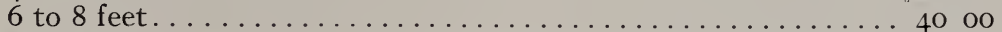

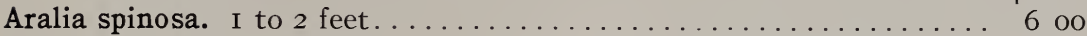

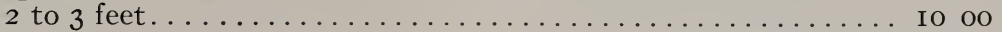

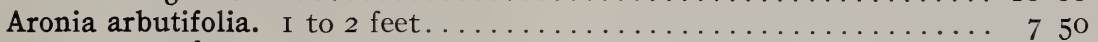

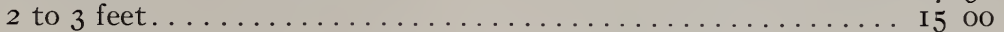

nigra. I to 2 feet. . . . . . . . . .

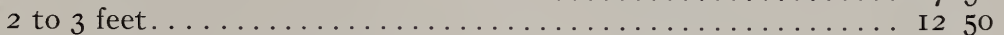

Azalea arborescens. 6 to 12 inches

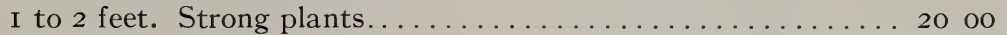

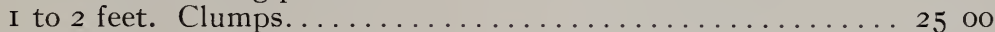

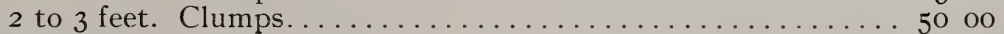

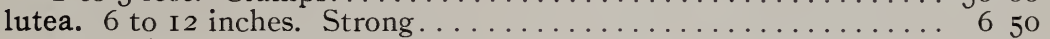

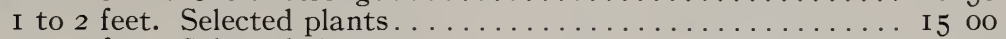

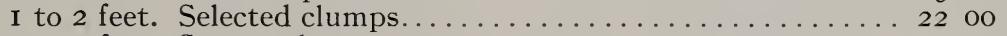

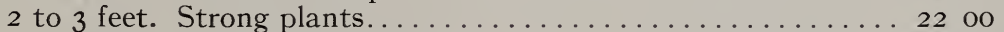

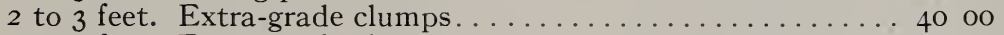

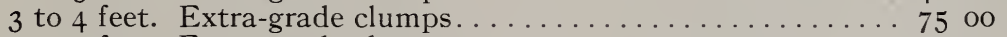

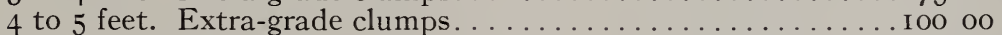

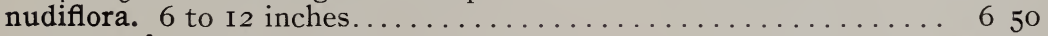

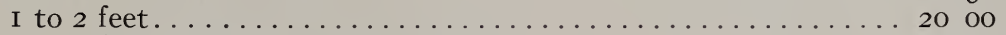

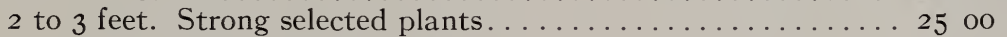

2 to 3 feet. Extra-grade clumps.......................... 40 oо

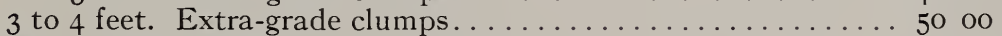

Vaseyi. 6 to 12 inches

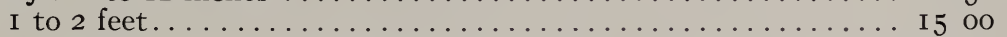

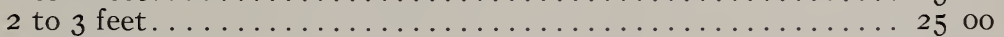

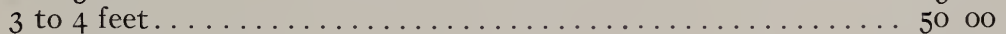

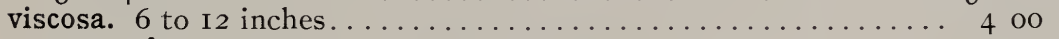

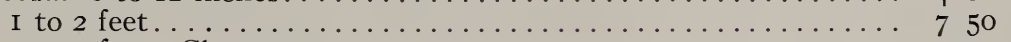

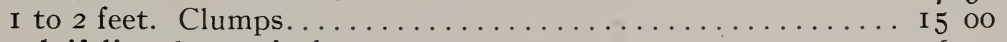

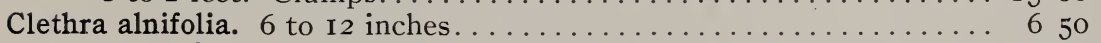

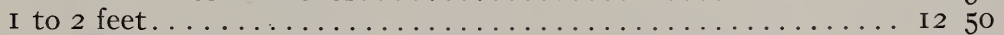

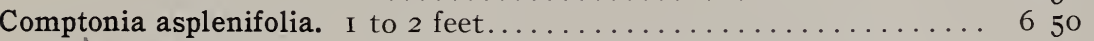

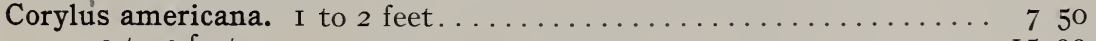

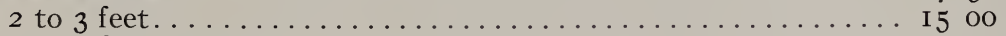

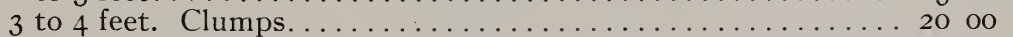

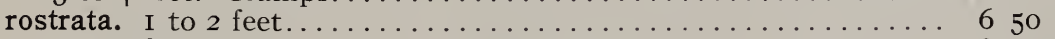

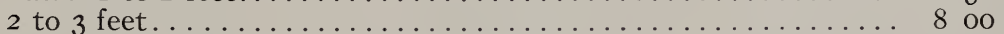

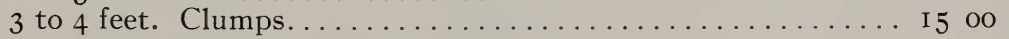

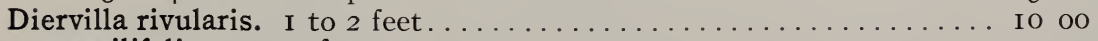

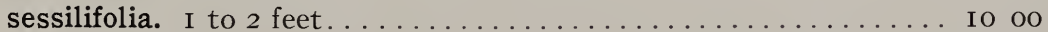

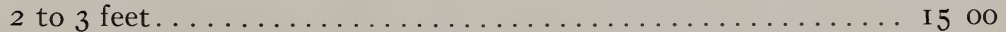

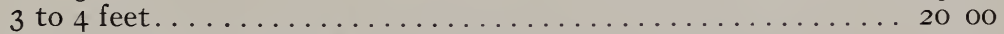

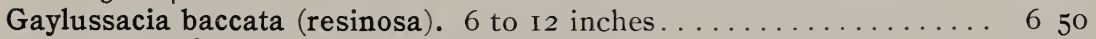

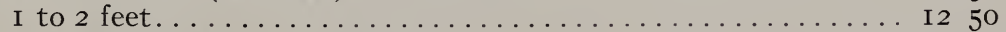

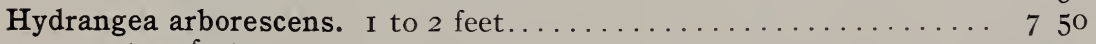

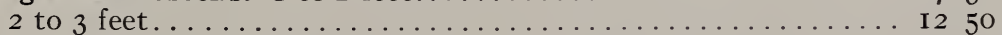

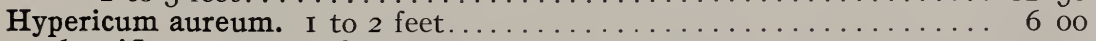

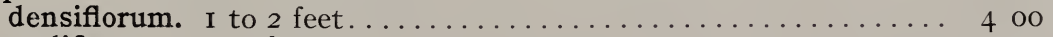

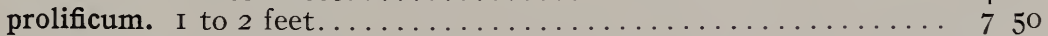

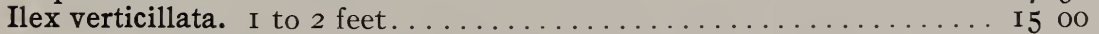

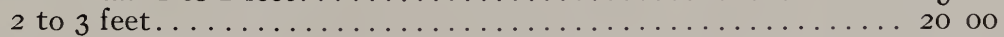

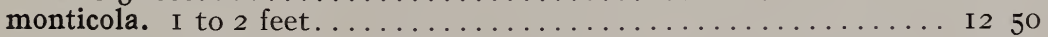

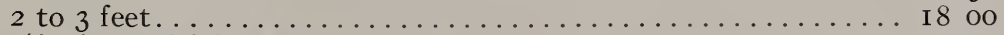

Lyonia (Andromeda) ligustrina. I to 2 feet $\ldots \ldots \ldots \ldots \ldots \ldots \ldots \ldots \ldots$ I 2 50

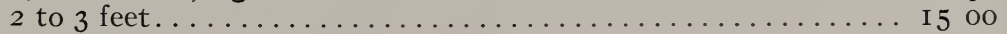

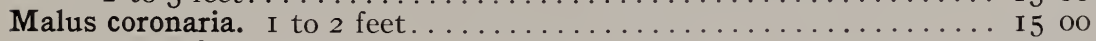

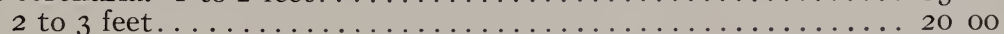

150 OO

350 00

5000

8000

6000

I 2500

6000

IOO 00

5000

I 5000

20000

5000

I 2500

20000

20000

350 00

60000

50 OO

17500

20000

350 OO

40000

50 OO

I 2500

20000

40000

2500

6000

I 3000

5000

IOO 00

$50 \quad 00$

6000

I 2500

17500

5 on

75 OO

I 2500

8000

$50 \quad 00$

5000

10000

50 OO

2000

6500
IOO 00 


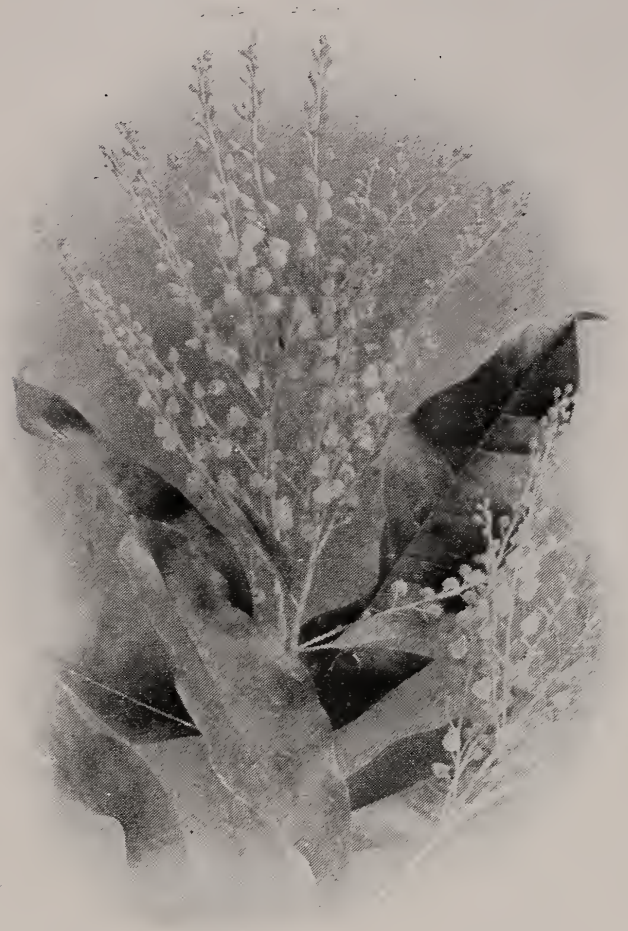

Oxydendrum arboreum
DECIDUOUS TREES AND SHRUBS,
continued
Menziesia pilosa. I to 2 Ico
feet. .............. $\$ 7$ 50 $\$ 6000$

Oxydendrum arboreum.

6 to 12 inches ... 5 oo 35 oo

I to 2 feet ...... $850 \quad 7500$

2 to 3 feet ...... I2 50 I00 oo

3 to 4 feet ......2 20 oo I75 oo

4 to 6 feet ........ 35 oo

6 to 8 feet ......5 50 oo

Rhus copallina. 6 to I2

inches. Seedlings.

Lots of 5,000 and

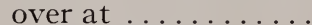

$\ldots \ldots 750 \quad 5000$

2 to 3 feet ...... I5 oo I25 OO

typhina. 2 to 3 feet . I 5 oo

Robinia hispida. I to 2

feet .......... I2 50 I00 00 2 to 3 feet ...... I5 Oo

viscosa. 2 to 3 feet .. I 250

3 to 4 feet ......2 20 oo

Rosa carolina. I to 2

feet.......... 5 oo

Sambucus canadensis. 2

to 3 feet ........ 5 oo

racemosus. 2 to 3 feet............ Io oo

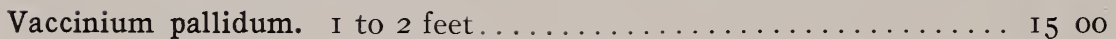

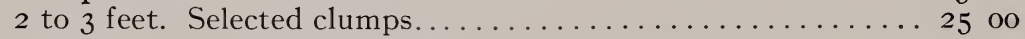

stamineum. I to 2 feet. Strong plants............... I2 50

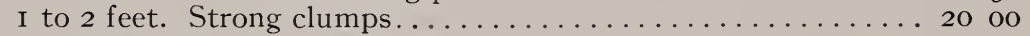

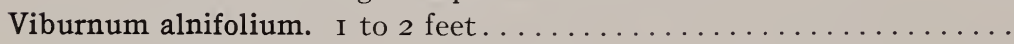

cassinoides. 2 to 3 feet. Strong plants................ Io oo

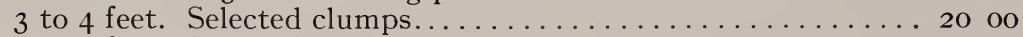

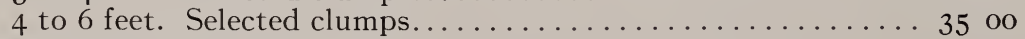

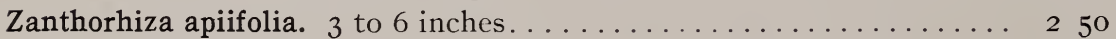

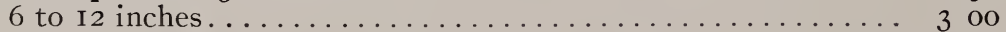

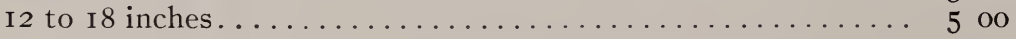

I25 00

IOO 00

I 75 OO

3500

\section{VINES}

Ampelopsis Engelmannii. Rooted cuttings ............... I5 oo

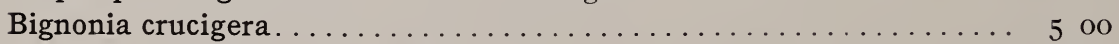

Clematis virginiana. Strong plants................... Io oo

Lonicera japonica Halliana. Light, for lining out . . . . . . . . . . . .

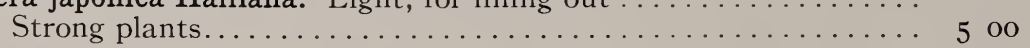

3500

80 oo

2500

Rubus hispidus. Splendid ground-covering plart............ 4 oo

canadensis. 2 to 3 feet. Strong plants ............... 5 оо

IO OO

2000

3000

Vaccinium macrocarpon. Cranberry ................. 4 oo 4000

35 oo

40 oo

2000

\section{EVERGREEN GROUND-COVERING PLANTS}

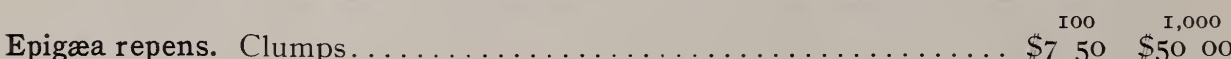

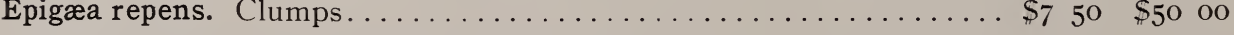

Galax aphylla. Extra-strong selected clumps............. 6 oo 45 оо

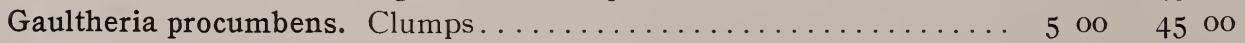

Mitchella repens. Clumps...................... 8 oo 6о о

Shortia galacifolia. Strong plants and light clumps.......... 8 oо 60 оо 


\section{NATIVE EVERGREEN TREES AND SHRUBS}

Abies Fraseri. 6 to I2 inches. Seedlings .................. \$I

Kalmia angustifolia. Nice stocky plants...............\$10 oо 90 oо

latifolia. 3 to 6 inches. Strong seedlings............... 4 oo 2500

6 to $\mathrm{I} 2$ inches. Strong stocky plants.................... 8 oo 70 oo

I to 2 feet. Strong bushy plants.................. 20 oo I75 oo

I to 2 feet. Selected clumps........................ 35 oo 300 oo

2 to 3 feet. Selected clumps..................... . . 65 oo 500 oo

3 to 4 feet. Selected clumps..................... . 90 oo 800 oo

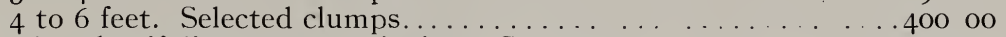

Leiophyllum buxifolium. 6 to 12 inches. Clumps.......... I2 o0 100 oo

I 2 to 18 inches. Clumps....................... I5 OO I25 Oo

prostratum. 2 to 4 inches. Extra-nice clumps ............. 55 oo 40 oo

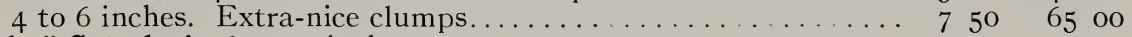

Leucothoë Catesbæi. 6 to 12 inches....................... 50 oo

I to 2 feet. Selected stocky plants................ I2 50 IOO

I to 2 feet. Well-furnished clumps.................. 25 oo 22000

2 to 3 feet. Well-furnished clumps................. 40 oo 350 oo

Pieris (Andromeda) floribunda. I I/2 to 2 feet. Selected clumps.......

2 to 3 feet. Selected clumps...................... I40 oo I250 O0

Seeds at $\$ 2.50$ per pound.

Rhododendron carolinianum. 3 to 6 inches. Seedlings.......... . $650 \quad 50$ oo

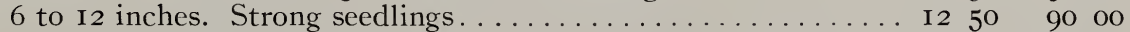

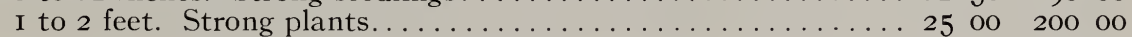

I to 2 feet. Strong selected clumps.................. 60 oo 500 oo

2 to 3 feet. Strong selected clumps................. I00 oо 900 oo

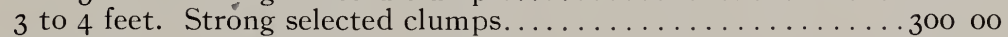

4 to 5 feet. Strong selected clumps.................. 400 oo

catawbiense. 3 to 6 inches. Select seedlings; well rooted. . . . . 5 oo

6 to 12 inches. Select seedlings; well rooted............ I2 50

I to 2 feet. Strong selected plants............... 35 oo

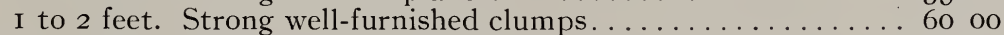

2 to 3 feet. Strong well-furnished clumps.............. 85 oo

3 to 4 feet. Strong well-furnished clumps.............. 250 oo

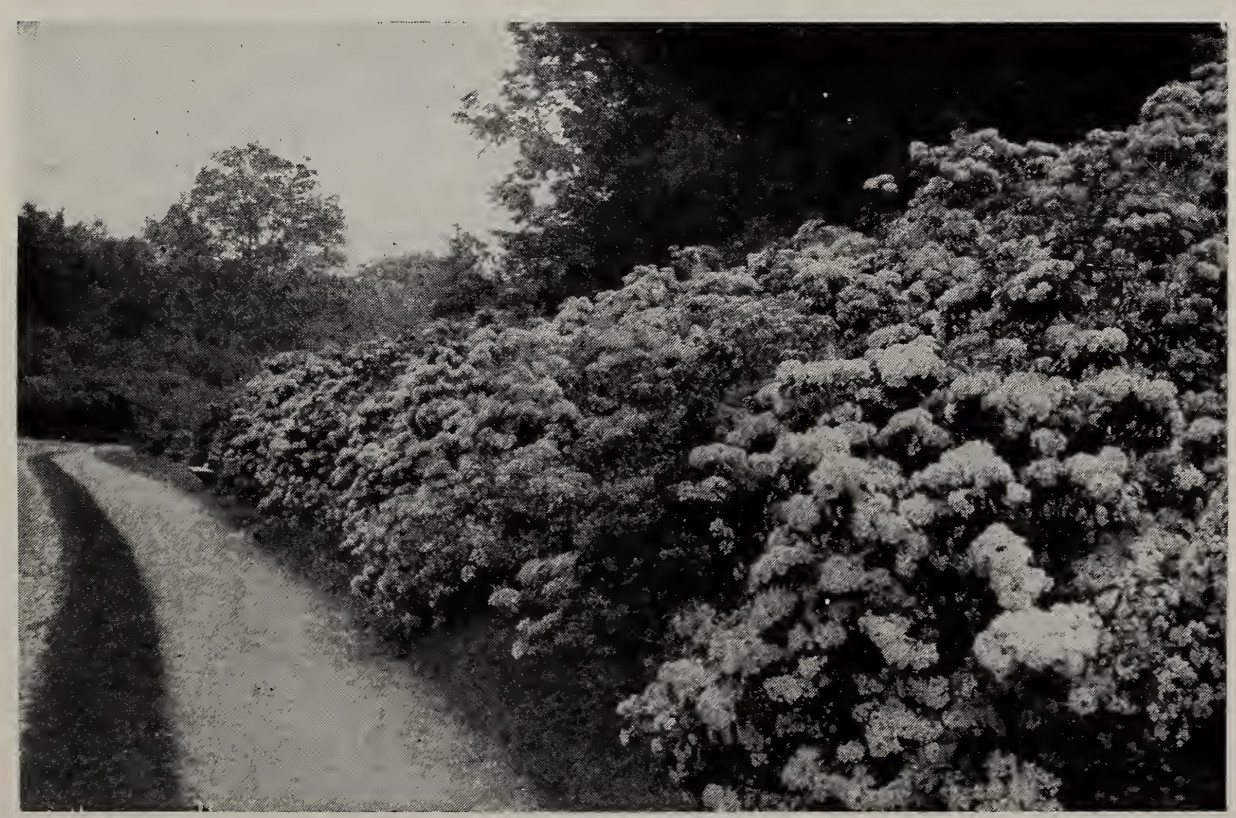

Kalmia latifolia 


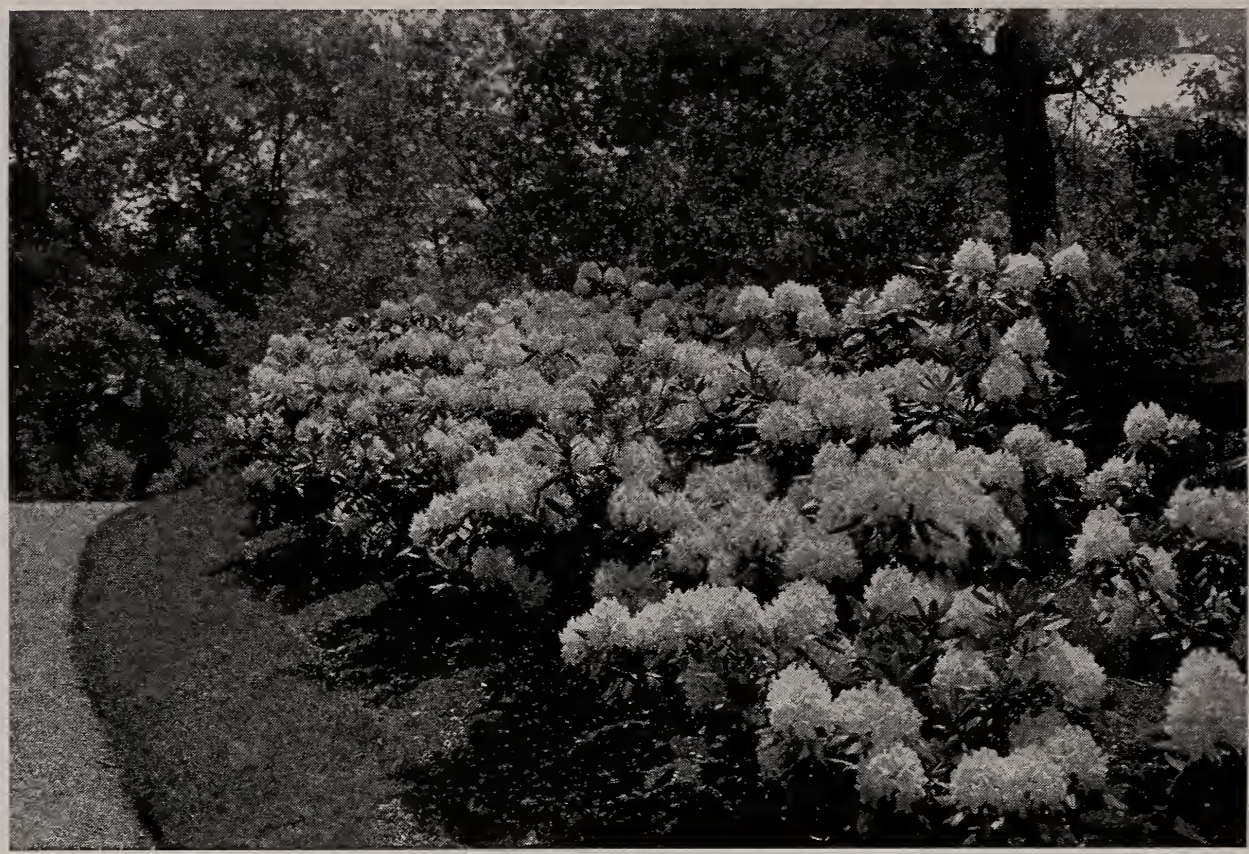

Planting of Rhododendrons

NATIVE EVERGREEN TREES AND SHRUBS, continued IOo

Rhododendron maximum. 3 to 6 inches. Seedling stock......... \$3 50 6 to 12 inches. Strong selected plants................. Io oo

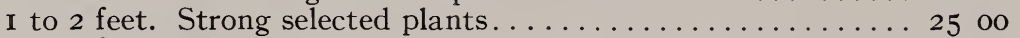

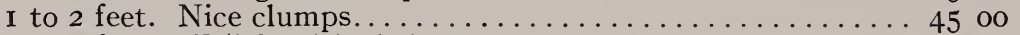

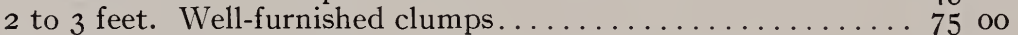

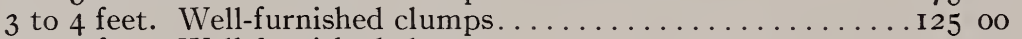

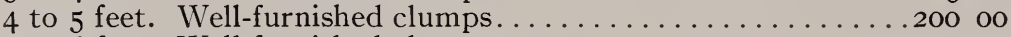

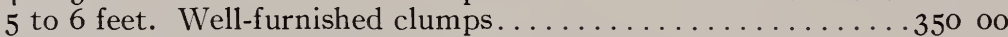

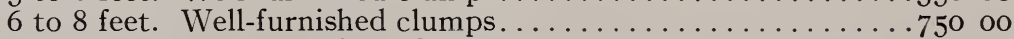

Tsuga canadensis. 6 to I 2 inches. Seedlings. Splendid root system...

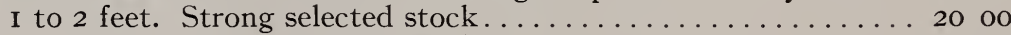

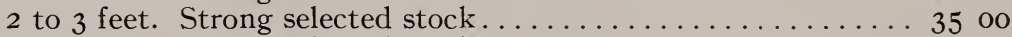

3 to 4 feet. Strong selected stock................. 75 oo

4 to 6 feet. Strong selected stock..................... I 5 . o o

6 to 8 feet. Strong selected stock. . . . . . . . . . . . . . 250 oo

caroliniana. 6 to I2 inches. Strong selected seedlings.......... I5 oo

I to 2 feet. Strong selected stock, balled and burlapped...... 75 oo

2 to 3 feet. Strong selected stock, balled and burlapped...... I 25 oo

3 to 4 feet. Strong selected stock, balled and burlapped . . . . . . . 200 oo

4 to 5 feet. Strong selected stock, balled and burlapped . . . . . . 300 o0

5 to 6 feet. Strong selected stock, balled and burlapped . . . . . . 400 oo

6 to 8 feet. Strong selected stock, balled and burlapped........500 00

Seed at $\$ 7.50$ per pound.

\section{HERBACEOUS PLANTS}

Aralia quinquefolia. Ginseng. I-year plants............. 3 oo

2 -year plants.................................... 4 oo

3 -year plants............................ 6 oo

Seeds.................................. 50

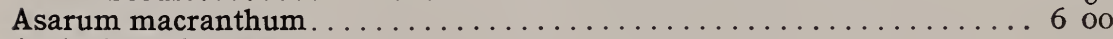

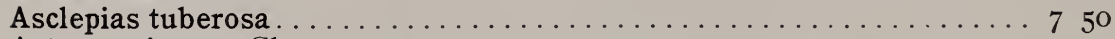

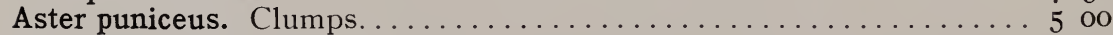

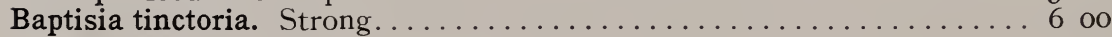

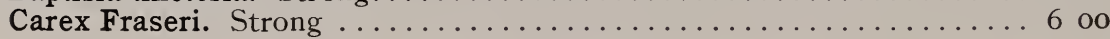


HERBACEOUS PLANTS, continued

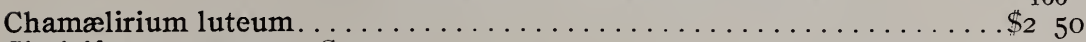

Cimicifuga racemosa. Strong........................

Cypripedium acaule. Strong $\ldots \ldots \ldots \ldots \ldots \ldots \ldots \ldots \ldots \ldots \ldots$ oo Light................................... 4 oo

pubescens. Strong.......................... 7 oo

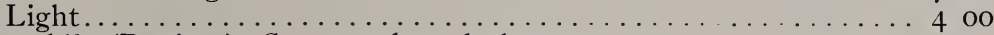

spectabile (Reginæ). Strong selected plants............... 9 oo

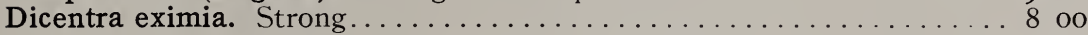

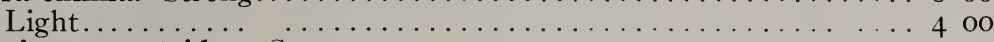

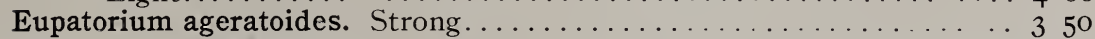

Erythronium americanum ................. I 50

FERNS, NATIVE. Special price on large orders.

Adiantum pedatum. Clumps.....................6 $50 \quad 50$ oo

Aspidium acrostichoides. Strong................... 5 oo 30 oo

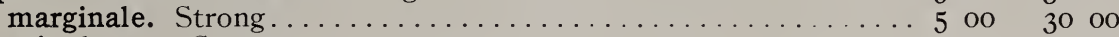

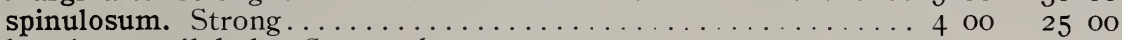

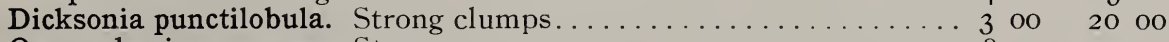

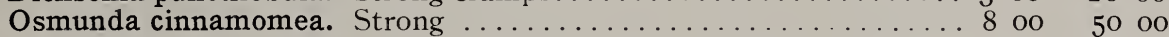

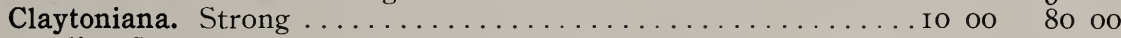

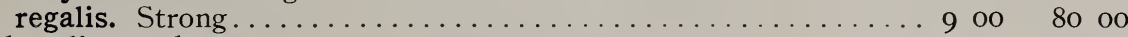

Polypodium vulgare . . . . . . . . . . . . . . . . . . . . 5 оо

Pteris aquilina...................... 5 oо

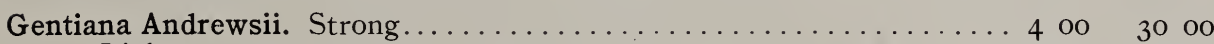

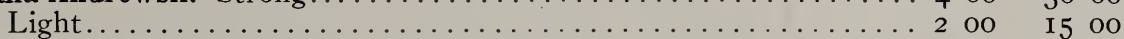

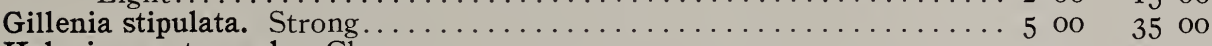

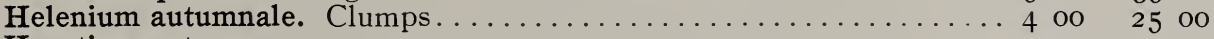

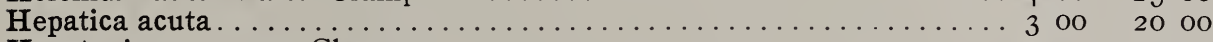

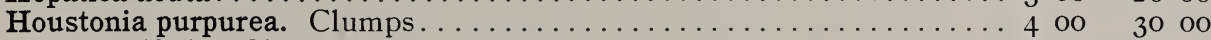

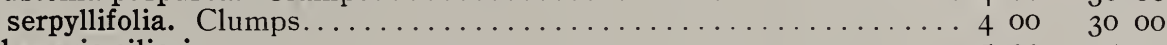

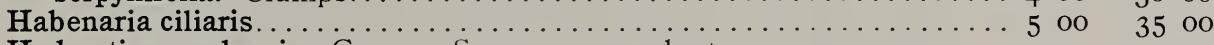

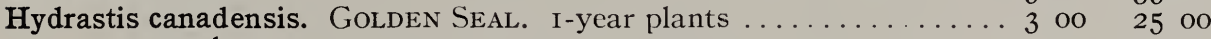

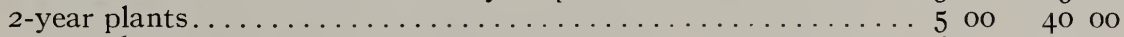

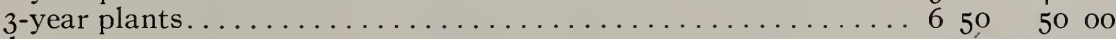

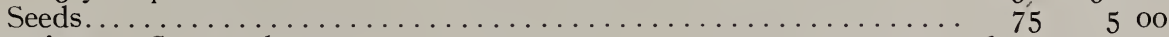

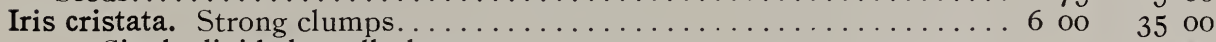

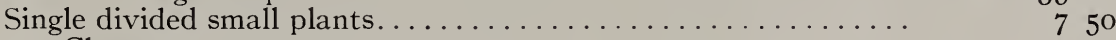

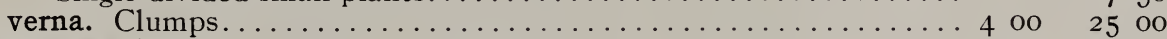

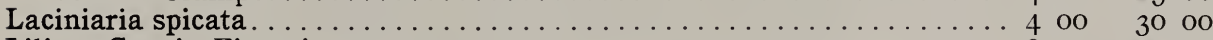

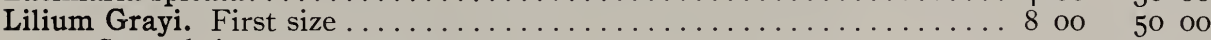

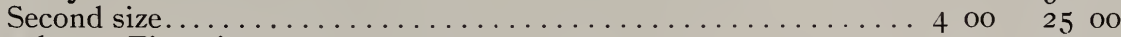

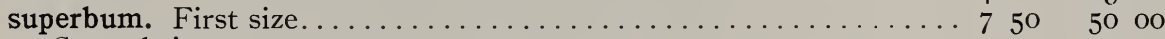

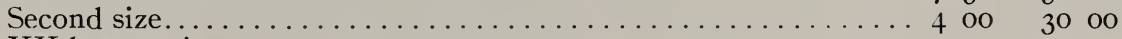

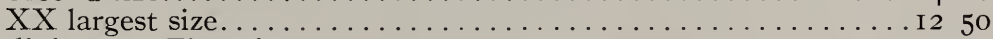

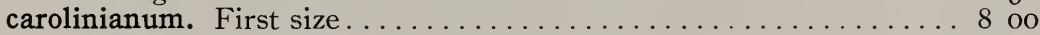

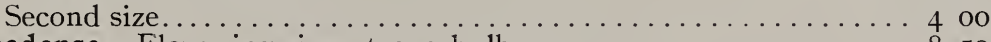

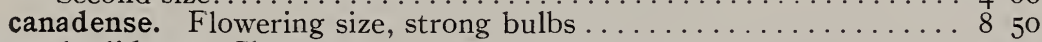

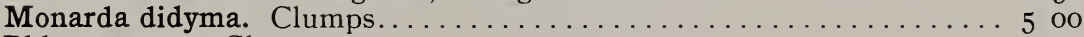

Phlox reptans. Clumps............................ 4 oo

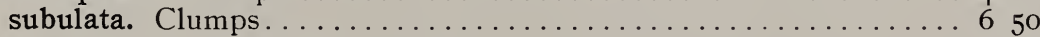

Polygonatum commutatum. Strong . . . . . . . . . . . . . . 4 oo

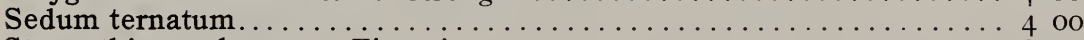

Sten anthium robustum. First size . . . . . . . . . . . . . . . . . . 8 oo

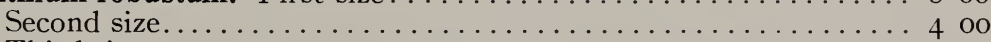

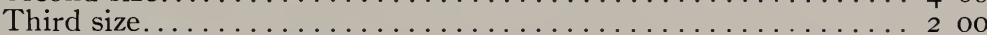

Trillium erectum album. Strong . . . . . . . . . . . . . . . 4 oo

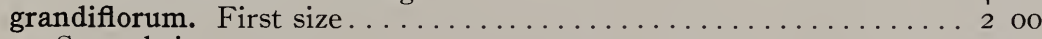

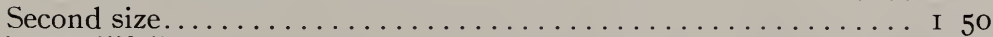

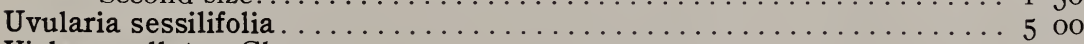

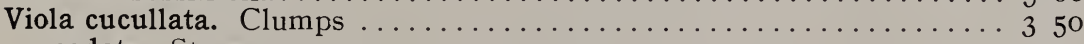

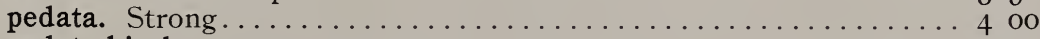

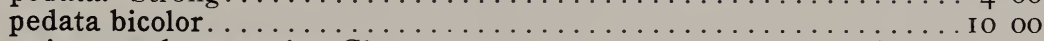

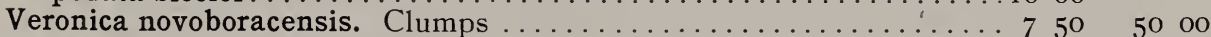

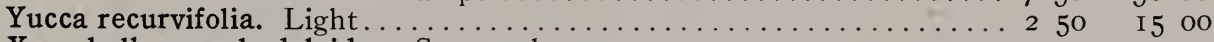

Xerophyllum asphodeloides. Strong clumps . . . . . . . . . . . . 5 oo 30 oo 
Landscape Architects, Nurserymen, and large planters will find my stock particularly suited to their requirements

\section{MY SPECIALTIES:}

Andromeda floribunda, Azaleas Rhododendrons, Kalmias, Oxydendrum Leucothoe Catesbaei Tsugas, and Zanthorhiza

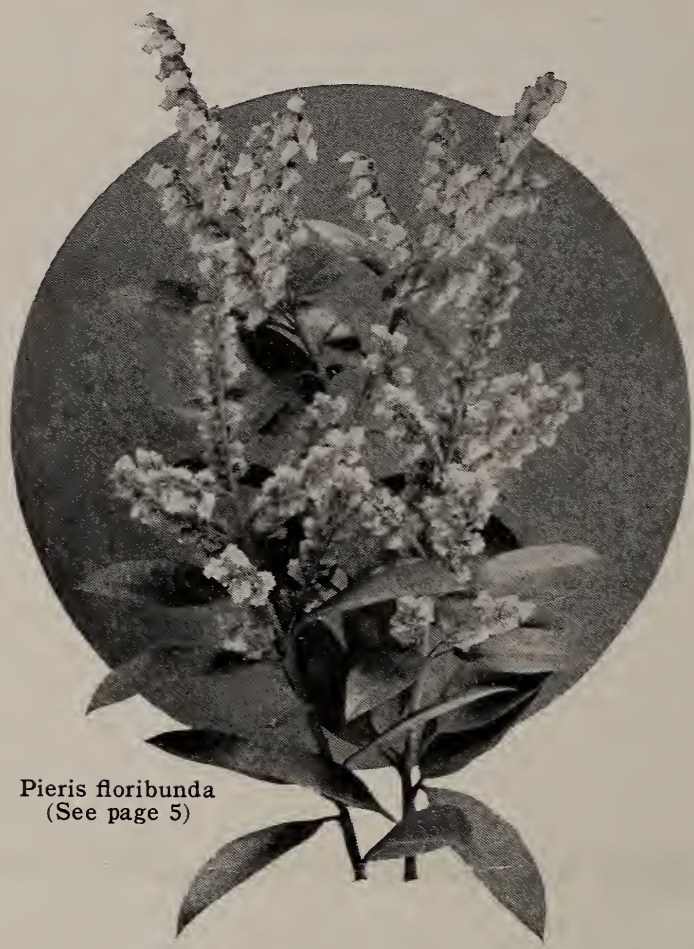

Your correspondence is invited and your orders will be given good attention. First come is first served, Always state when burlapping is desired, letting me have your list of requirements as quickly as possible to insure getting the stock in good time for planting.

\section{E. C. ROBBINS}

PINEOLA, Avery Co. : NORTH CAROLINA 